\title{
Desarrollo de la nefrectomía durante el siglo XIX y su introducción en España
}

\author{
Pérez Albacete M, Tornero Ruiz J, López Cubillana P. \\ Servicio de Urología. Hospital Universitario Virgen de la Arrixaca. Murcia. \\ Actas Urol Esp. 2008;32(2):172-178
}

\section{RESUMEN}

DESARROLLO DE LA NEFRECTOMÍA DURANTE EL SIGLO XIX Y SU INTRODUCCIÓN EN ESPAÑA

Introducción y objetivos: Tras la primera nefrectomía lumbar realizada por el Dr. Simon en 1869, la técnica quirúrgica quedó perfilada y el procedimiento fue seguido por numerosos cirujanos en controversia inicial entre la utilización de la vía de acceso intra o extraperitoneal. Efectuamos una revisión acerca de la evolución llevada por la operación en el siglo XIX y sobre quienes iniciaron la intervención en nuestro país.

Material y Métodos: Hemos revisado los libros de historia de la Urología, los capítulos de cirugía en los tratados clásicos de la especialidad, las comunicaciones a los congresos españoles de Medicina, hasta principios del siglo XX y las operaciones de nefrectomía publicadas en revistas médicas de la época.

Resultado. Analizamos diferentes estudios estadísticos aparecidos en este periodo sobre los resultados de la operación, la disminución de la mortalidad en las nefrectomías realizadas por vía extraperitoneal demostró pronto su ventaja. Localizamos 35 extirpaciones renales por medio del acceso lumbar, efectuadas en España en el último tercio del siglo XX, tres del Dr. Federico Rubio y Galí en Madrid, en Barcelona de los urólogos Dr. Víctor Azcárreta Colán y José Pagés y de los cirujanos Drs. Esquerdo y Salvador Cardenal, del sevillano Dr. Antonio Solano y del urólogo canario Dr. José Hernández Pérez, quienes fueron los pioneros de la intervención en nuestro país.

Conclusión: La introducción de la nefrectomía por vía lumbar en España fue tardía en relación con el resto de Europa, debido al atraso secular que arrastrábamos, únicamente cuando las condiciones generales de los quirófanos, tanto de anestesia como de asepsia y de utillaje quirúrgico, permitían poder abordar con garantía de éxito la operación, los principales cirujanos comenzaron a realizarla.

Palabras clave: Historia de la Urología. Nefrectomía. siglo XIX. Nefrectomía en España.

\begin{abstract}
DEVELOPMENT OF NEPHRECTOMY DURING THE NINETEENTH CENTURY AND ITS INTRODUCTION INTO SPAIN

Introduction and objectives: After first nephrectomy lumbar made by Dr. Simon in 1869, the surgical technique was established and the procedure access was followed by several surgeons with initial controversy between the use of the intra or extraperitoneal access. We carried out a review about the evolution of this surgical procedure among XIX century and of those who were pioneers with this Kind of technique in our country.

Material and methods: We have reviewed books of history of the Urology, the chapters of surgery in classic urology volumes and the oral presentations to the Spanish Medical congresses, until beginning of XX century and the published nephrectomy procedures in medical literature of that time.

Results: We analyzed different statistical studies appeared at the time about results of the operation, the decrease of mortality in the nephrectomies made by extraperitoneal access demonstrated its advantage soon. We found out 35 kidney removal performing the lumbar access, carried out in Spain in the last third of the XX century, three by Dr Federico Rubio and Gali in Madrid, in Barcelona by the urologist Dr. Víctor Azcárreta Colán and Jose Pagés and by the surgeons Drs. Esquerdo and Salvador Cardinal, from Sevilla, Dr Antonio Solano and the canary urologist Dr. Jose Hernández Perez, who were pioneers performing this procedure in our country.

Conclusions: The introduction of nephrectomy by lumbar access in Spain was delayed in relation to the rest of Europe, due to the secular delay that we dragged, only when adequate conditions of the operating rooms, as much as anaesthesia and asepsis and surgical devices tools, guaranteed the success operation, the main surgeon begun to perform it.
\end{abstract}

Keywords: History of the Urology. Nephrectomy in the 19th century. Nephrectomy in Spain. 
Hasta el siglo XIX las operaciones en las afecciones renales se habian limitado a desbridamientos de abscesos perinefríticos y a la exploración de alguna herida de la región lumbar pero, tras la implantación de la anestesia en 1846, se amplió el campo de la cirugía y se discutía sobre la oportunidad de practicar una "talla" al riñón para extraer los cálculos de su interior al igual que se hacía con los de la vejiga; se tenían dudas sobre si se podría sobrevivir con un solo órgano al extirpar el otro, aunque ya se conocía desde el siglo XVII, por autopsias realizadas por Berenguer de Carpio, Riolano, Bellini y otros, la existencia de personas monorrenas, congénitas o por destrucción de uno de los órganos; se temía una hemorragia incoercible durante la operación de nefrectomía, por lo que se llevó a cabo en animales, aunque en alguna ocasión los cirujanos generales, en el transcurso de intervenciones abdominales por vía transperitoneal $\mathrm{y}$, por error de diagnóstico, habían extraído algún riñón sin que se hubiera producido; pero el peligro de una infección al manipular el peritoneo conllevaba un alto riesgo de muerte por complicaciones sépticas, lo que frenaba su ejecución.

El profesor de cirugía de Heidelberg, Gustav Simons (1824-1876) (Fig. 1), tuvo el mérito de realizar, en 1869, la primera nefrectomía por vía lumbar subcostal, en una paciente de 46 años con una

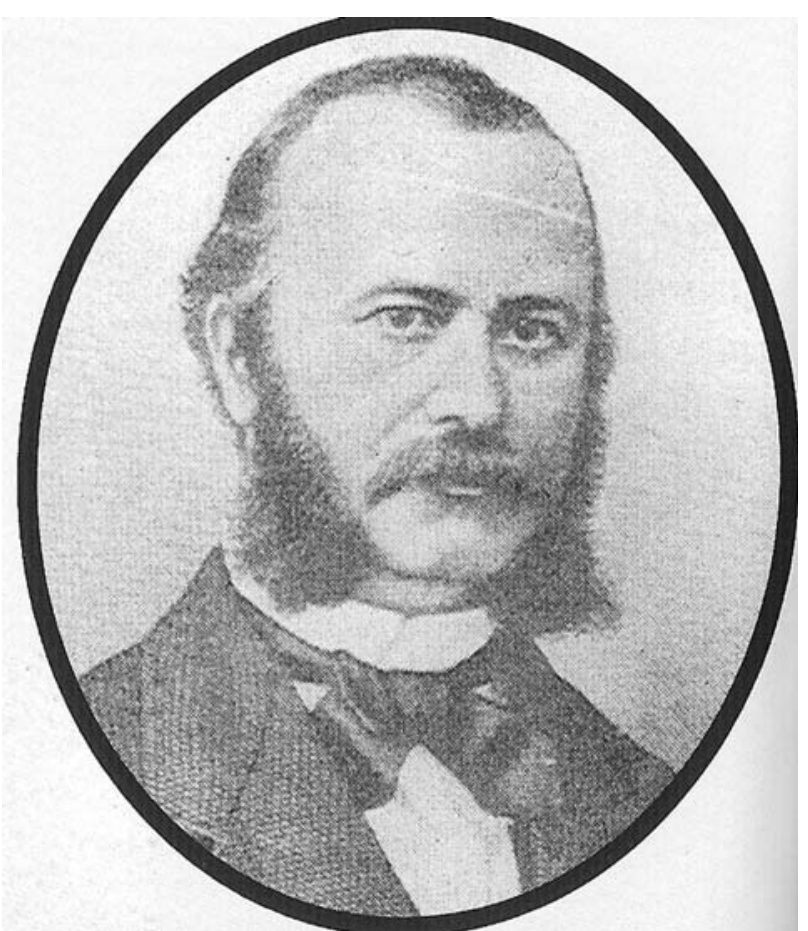

FIGURA 1. Dr. Simons (1824-1876) profesor de cirugia en Heidelberg. fistula uretero-vaginal y uretero-cutánea, secundaria a una intervención ginecológica, a la que había intervenido para solucionársela en tres ocasiones previas sin resultado y consideró que la única alternativa para resolver el problema era la extirpación renal; con objeto de eludir las maniobras intraperitoneales, se preparó concienzudamente, estudió cuidadosamente la anatomía de la región en la sala de disección para ver la accesibilidad a la glándula sin necesidad de abrir el peritoneo, efectuó la operación primero en quince perros con objeto de evaluar las dificultades que ofrecía el acceso y después en cadáveres para familiarizarse con la vía lumbar subcostal que diseñó, la disección del órgano y de su pedículo vascular y, una vez consciente de su viabilidad, la llevó a la práctica; realizó la nefrectomía con anestesia clorofórmica en cuarenta minutos, sin contratiempo y la enferma curó tras sobreponerse de un postoperatorio de seis meses por complicaciones de íleo paralítico, neumonía e infección (Fig. 2); en 1871 hizo la segunda en una

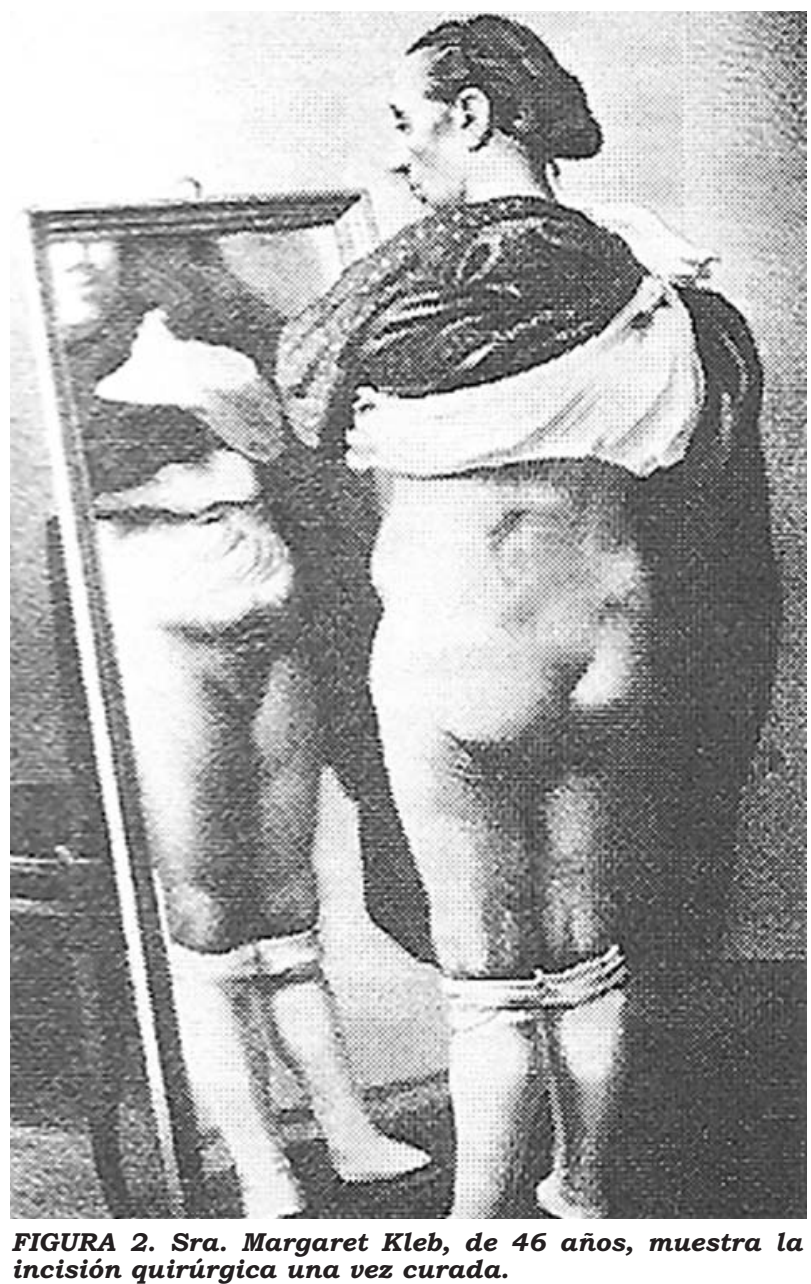


paciente diagnosticada de una calculosis renal complicada, que falleció en el postoperatorio tardío de un cuadro séptico ${ }^{1,2}$.

Tras la divulgación de la técnica preconizada por Simons, numerosos cirujanos se apresuraron a plantear la indicación de la extirpación renal, el principal problema consistía en saber cuál era el riñón enfermo, conocer la función del contralateral y asegurar que, con la operación, se lograría la curación por lo que se limitaban a intervenir aquellos casos en los que se presentaban cuadros dolorosos, por abscesos, tumoraciones palpables o historia clara de litiasis en un lado, unido a la habilidad clínica, a la exploración manual y finura de la palpación y en ocasiones se llegó a confirmar el diagnóstico con la punción del órgano afecto y extraer orina en las hidronefrosis o pus en las pionefrosis, lo que unido a la intrepidez de los grandes cirujanos hizo que paulatinamente se afrontase su realización, fueron los pioneros en Alemania, Czerny e Israel, en Francia Le Dentu, Guyon, Tuffier y Albarrán, en Inglaterra, Morris y Newman(1).

Comienzan a describirse en las publicaciones periódicas de la época casos de nefrectomías por vía lumbar y estadísticas comparativas de los resultados postoperatorios entre el abordaje intra o extraperitoneal, así el Dr. Brodeur recoge en París, en 1886, en su obra, De la intervención quirúrgica en las afecciones del riñón, citada por el Dr. Más y Soler ${ }^{3}$ (1847-1902), de 327 intervenciones efectuadas hasta ese momento, sin diferenciar patologías, 125 fueron lumbares, con un $62,4 \%$ de

(1)Czerny (1842-1916), austriaco de nacimiento desarrolló toda su carrera en Heidelberg, utilizó la vía intercostal y realizó la primera nefrectomía parcial por tumor en 1887.

Israel, James (1848-1926), urólogo berlinés de origen judío, fundador de la especialidad en Alemania y gran autoridad urológica.

Le Dentu, Jean (1841-1926), profesor de la clínica de cirugía del Hôtel Dieu de París, en 1886 publicó su "Técnica de la nefrectomía" y realizó junto con Albarrán la primera nefroureterectomía por tumor, en 1889. Félix Guyon (1831-1920), profesor de la clínica urológica del Hospital Necker de París y considerado como el creador de la especialidad de Urologia.

Tuffier, Teodoro (1857-1929), cirujano urólogo de los Hospitales La Pitie y Lariboisière de París, destacó por su contribución a la cirugía renal. Sir Henry Morris (1844-1926), profesor de la Clínica quirúrgica de la Universidad de Edimburgo, en 1885 publicó en Londres el libro "Cirugía de las enfermedades del riñón".

Albarrán, Joaquín (1860-1912), hispano cubano de nacimiento, realizó toda su carrera en el Hospital Necker de París, donde fue jefe del servicio de Urología, realizó una nefrectomía parcial en 1898 y colaboró con Le Dentú en la primera nefroureterectomía por tumor.

Newman, David, excelente cirujano de Glasgow. curaciones, frente a 110 transabdominales con un $50 \%$ de supervivencia; el Dr. Mollá Rodrigo (1862-1930) ${ }^{4}$ nos presenta una serie de operaciones por tumor renal de cirujanos europeos anteriores a 1890, en las cuales el número de fallecidos la no baja del $60 \%$ tanto en el niño como en el adulto; el Dr. Guyón (1831-1920)5 , en 1892, recoge los resultados de los Drs. Bureau, con un $22 \%$ de nefrectomías extraperitoneales y un $62 \%$ de intraperitoneal y de Samuel Warnolts-Gross (1805-1884), profesor de cirugía del Medical College de Jefferson en Filadelfia, que sobre 234 intervenidos a través del abdomen tiene 109 $(46 \%)$ curados y 104 fallecidos y por vía extraperitoneal de 111 casos, cura a $70(63 \%)$ y tiene 41 defunciones.

El Dr. Juan de la Sota y Lastra, catedrático de Patología General en la Escuela de Medicina de Sevilla en una conferencia dada, el 5 de noviembre de 1891, sobre Extirpación total de riñón derecho. Consideraciones sobre la nefrectomía (Fig. 3$)^{6}$, tras presentar un caso de extracción renal transabdominal por una hidronefrosis obstructiva por litiasis seguida de curación, realizada en 1890, efectúa un excelente estudio sobre el desarrollo y evolución del procedimiento y una amplia revisión de la bibliografia publicada hasta esa fecha en libros, revistas, monografias nacionales y extranjeras y extrae un total de 440 nefrectomias llevadas a cabo y publicadas por 156 cirujanos

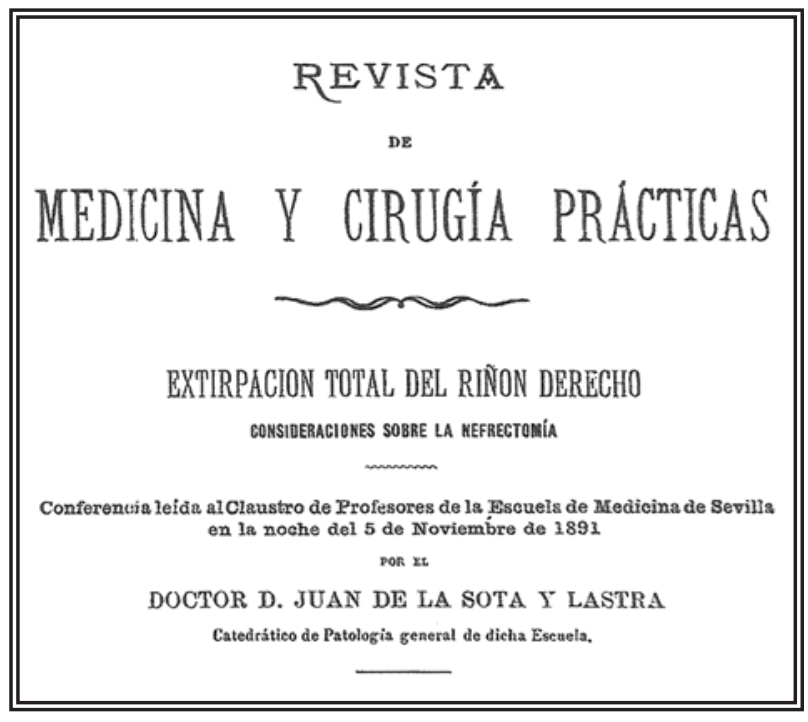

FIGURA 3. Conferencia del Dr. Sota y Lastra, sobre Extir pación total del riñón derecho. Consideraciones sobre la nefrectomía. Sevilla, 1891. 
diferentes (Fig. 4), de ellas únicamente encuentra hechas en España, por vía lumbar tres del Dr. Rubio y Gali en Madrid, y una en Sevilla por el Dr. Antonio Salado. Las mayores estadísticas que encuentra son la citada anteriormente del Dr. Gross y la presentada por el Dr. Henry Morris, con 141casos. Del total de las operaciones, fueron intraperitoneales 206, con 93 curaciones (45\%) y 113 defunciones y por vía lumbar de 201, curaron $125(62 \%)$ y fallecieron 76 , las causas del exitus fueron shock, hemorragias y peritonitis en las abdominales y la de sepsis, anuria, convulsiones, vómitos y uremia en las extraperitoneales (Fig. 5).

La polémica sobre la vía de elección se mantuvo hasta finales del siglo, en que la mayoría de los cirujanos aceptaba el procedimiento intraperitoneal en los casos de grandes masas y la vía lumbar en los cuadros infecciosos y de pequeños tumores; fue el Dr. James Israel ${ }^{7}$, tras exponer sus resultados en 1891, en los que disminuyen las cifras de mortalidad postoperatoria al 16\%, en 81 pacientes intervenidos por vía lumbar y aún la logra descender al 10\% en el año 1900, gracias a su habilidad y depurada técnica quirúrgica y quien, con su gran autoridad, recomendó huir de

Para terminar lo que me habia propuesto en esta segunda parte de mi conferencia, debo deciros los nombres de los operadores que conozco han ejecutado la operación; quitando el primero que la practicó y á quien ya os he nombrado, adoptaré el orden alfabético para enumerar los sucesivos. Y son estos:

Angelini, Andersson, Bardenheur, Bantok, Bryant, Barker, Bottini, Barwell, Beck, Billroht, Bouvell, Burgeus, Bassini, Czerny, Clements, Couper, Callender, Crane, Clevelland, Clark, Culling, Cabot, Craus, Credi, Daudrige, D'Antona, De Vecchi, Dunreicher, Duncan, Dawsson, Durtraut, Day, Dunning, Elder, Esmarch, Feralino, Freuch, Goodridge, Fucterbock, Goodlee, Golding-Bird, Gowdell, Getz, Gilmore, Gaadneer, Gaillard Thomas, Gardner, Gross, Hicquet, Holmes, Haward, Hulke, Hearth, Heusner, Hahn, Israel, Ingalls, Jones, Joverts, Jurie, Jessop, Kœning, Kild, Ker, Keys, Kœling, Krambrals, Kuster, Laroroze, Lawsson Tait, Lange, Landeau, Lister, Lucas, Le Dentu, Landi, Leopold, Lance, Le Fort, Little, Langenbeck, Llody, Martin, Marcus Beck, Morris, Marsh, Mitehead, Mynter, May, Minckel, Madlau, Martini, Martell, Novaro; Navrati, Neuman, Ollier, O'Reilly, Panggi, Pepper, Palmer, Parker, Petersson, Purey, Peter, Penice, Polk, Péan, Polaillon, Roberts, Raffa, Ruves, Roddick, Rosemberger, Rossenbach, Rose, Richardsson, Raudon, Ressey, Rubio, Savage, Stoawell, Studsgaard, Sente, Sunley, Simón, Sonemburg, Seymour, Sorren, Spiegelberg, Schrram, Spencer-Wells, Stumsson, Sweussen, Salado, Thomas Smith, Thor-thon, Tschermicg, Thiersoh, Taylor, Tyfani, Von Bergman, Von Brun, Von Mandach, Van-Buren, Von Murat, Viglie, Vercilli, Weir, West, Williams, Wright, Walter, Zweifel y Urbinati.

FIGURA 4. Lista de cirujanos que habian realizado nefrectomias antes de 1890.

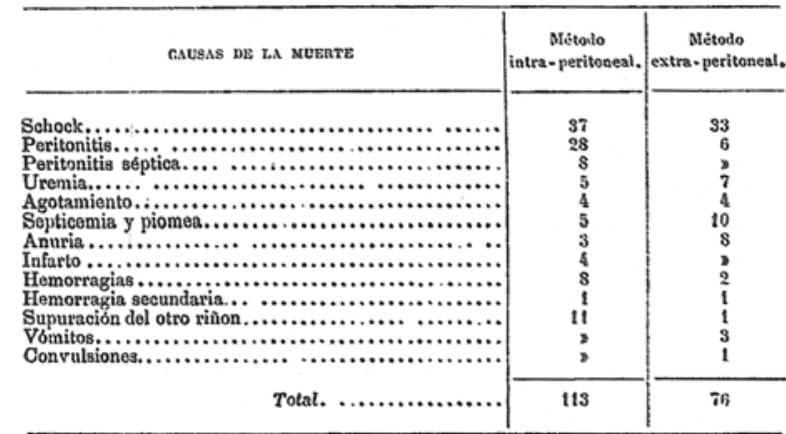

FIGURA 5. Causas del fallecimiento en las 189 operaciones.

abrir el peritoneo por el gran riesgo de infección y prolongar lo que fuera preciso la incisión lumbar en las intervenciones por extensos tumores.

En España fue el Dr. D. Federico Rubio y Gali (1827-1902) (Fig. 6), en el Instituto de Operatoria de Madrid, el primero en realizar una nefrectomía por vía lumbar, en 1874, seguida de una segunda, dato expuesto por el autor en una conferencia [citado por el Dr. Sota y Lastra en $1892^{6}$ y, entre otros, por Riera Palmero ${ }^{8}$ nuevamente en

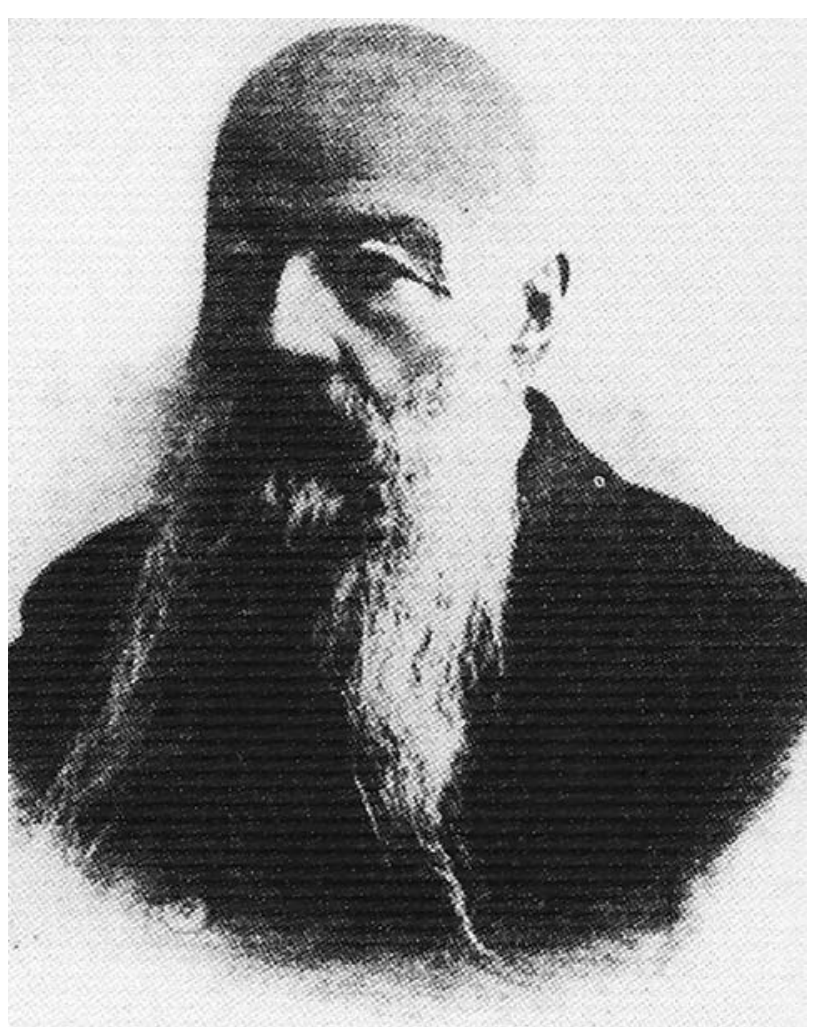

FIGURA 6. Dr. Federico Rubio Gali (1827-1902) fundador del Instituto de Operatoria en Madrid y quien realizó la primera nefrectomia por vía lumbar en España en 1874. 
1890 publica un caso en el que extirpa un riñón precedido de una punción percutánea aspirativa renal para confirmar el diagnóstico de pionefrosis, con resultado postoperatorio satisfactorio ${ }^{9}$.

El Dr. D. Álvaro Esquerdo ${ }^{10}$, cirujano del Hospital de la Santa Cruz y de San Pablo, de Barcelona, efectúa entre 1885 y 1887 dos nefrectomías extraperitoneales, una en un paciente con un quiste de riñón y la segunda en una ectopia renal con una evolución normal y restablecimiento total en ambas.

El Dr. Azcárreta Colán (1857-1937), urólogo de origen bilbaíno afincado en la Ciudad Condal, en 1890, lleva operadas dos nefrectomías por vía lumbar ${ }^{11}$ en pacientes diagnosticados de tuberculosis, con buen resultado y curación del proceso fimico de los enfermos; posteriormente, en 1898, en el Congreso Hispano Portugués de Cirugia y sus Especialidades Naturales ${ }^{12}$ (Fig. 7) amplia su estadística con cinco casos más desde el año 1892; en 1899 presenta su serie de nefrectomías por litiasis, en la publicación editada y dirigida por él, Revista Quirúrgica de Enfermedades de Vias Urinaria ${ }^{13}$ (de la que no se conoce la existencia de ejemplares) y en 1901 recoge en la Revista de Medicina y Cirugía Práctica (Fig. 8), su serie de cuatro casos de nefrectomía lumbar, por tumores de gran tamaño con buena evolución postoperatoria y una supervivencia de cinco años ${ }^{14}$.

\section{CINCO IEFRECTOMIAS POR TUBRRCULOSIS DEL RIÑON}

$$
\begin{gathered}
\text { POR EL } \\
\text { POCTOR AZCARRETA. }
\end{gathered}
$$

Es en vano que yo recuerde, puesto que es del dominio general, que la tuberculosis del riñon ocupa lugar preferente en las conquistas de la Cirugía moderna, que, á guisa de Alejandro 6 de Napoleón el Grande, sueña, y, por las trazas, parece que consigue, el Imperio universal, desde el momento que no existe para ella lugar sagrado, y que las vísceras más nobles son invadidas por el bisturí.

Hace pocos años, la tuberculosis del riñón no tan sólo era tributaria de la Patología interna, sino que constituía un terreno inexplorado para el diagnóstico. Comenzaron este bellísimo período de la Cirugía renal: Morris y Newman, en Inglaterra; Czerny é Israel, en Alemania; Le Dentu Guyon y Tuffier, en Francia; en España me cabe el honor de haber sido uno de los primeros en rendir á la humanidad el gran beneficio de librar algunos de sus miembros de una dolencia tan grave y tan radicalmente curable por la intervencion cruenta, como es la tuberculosis primitiva del riñón. Y es que el bacilo de Koch, que en otras vísceras, como en fortaleza inexpugnable, resiste, por desgracia, los embates de la Terapéutica, se rinde en el riñón al soberano poder de la Cirugía.

Desde el momento que la Clínica y la dnatomía patologica aseveraban, bajo la palabra de Bayle, Rayer, Trawellier, Morris, Newman y Guyon, que el bacilo de Koch puede anidarse largos años en un riñón, respetando el resto del organismo, debí surgir, como natural corolario, la idea de extirpar el 6 rgano enfermo, como se reseca un hueso 6 se extirpa un testículo tuberculoso.

FIGURA 7. Dr. Azcárreta, Cinco nefrectomias por tuberculosis, Congreso Hispano Portugués de Cirugia y sus especialidades, Madrid, 1898.
E1 Dr. Salvador Cardenal Fernández (18521927) (Fig. 9), cirujano del Hospital de Sagrado Corazón de Jesús de Barcelona, en la tercera edición de su Manual práctico de cirugía antiséptica ${ }^{15}$,

Resultados de la intervención quirúrgica en los tumores malignos del riñón.En la Revista de Ciencias Médicas de Barcelona, publica el Dr. Azcarreta las siguientes ideas :

El criterio acerca de las grandes operaciones modernas ha de pasar forzosamente por dos fases. Que el resultado inmediato de la nefrectomía en los tumores del riñón es por demás \&atisfactorio, pero que sus resultados lejsnos fuesen también excelentes, es una afirmación que fué preciso mantener in pectore, sujeta á una observación prolongada, para que transcurrido algún tiempo se pudiera procismar de qué modo aquellos enfermos que le presentaron agotados por las hematurias y los sufrimientos, y á quienes extirpo su riñón, gozan después de varios años de una salnd floreciente y perfecta.

Es un hecho indiscutible, que los tumores renales son una feliz y venturosa excepción, en medio de los desastres más 6 menos rápidos que deslnstran ls intervención quirúrgica en los neoplasmas de otras vísceras.

La clínica demuestra que la recidiva en los tumores renales, $\delta$ no se presenṫa, 6 es muy tardia. Podrá argüirse que los enfermos llevan pocos años desde la operación ; sin embargo, existen nefrectomizados hace nueve y diez años, período de tiempo suficiente pars poder decir que el resultado lejano de esta operación es altamente favorable á la humanidad. ¿A qué se debe este hecho de observación? A su juicio, entre las diversas explicaciones que se han propuesto, la única aceptable es el papel defensivo de la cáp sula fibrosa renal. Es evidente que si los ganglios del hilo están invadidos, la recidiva es rápida y segura, y pnesto que la clínica proclama que la enfermedad no retoña, gran número de veces, en pos de la nefrectomía, hay que admitir lógicamente que la propagación á los ganglios y á las venas es tardia.

FIGURA 8. Dr. Azcárreta, serie de cuatro casos de nefrectomía lumbar, por tumores Revista de Medicina y Cirugia Prácticas, 1901.

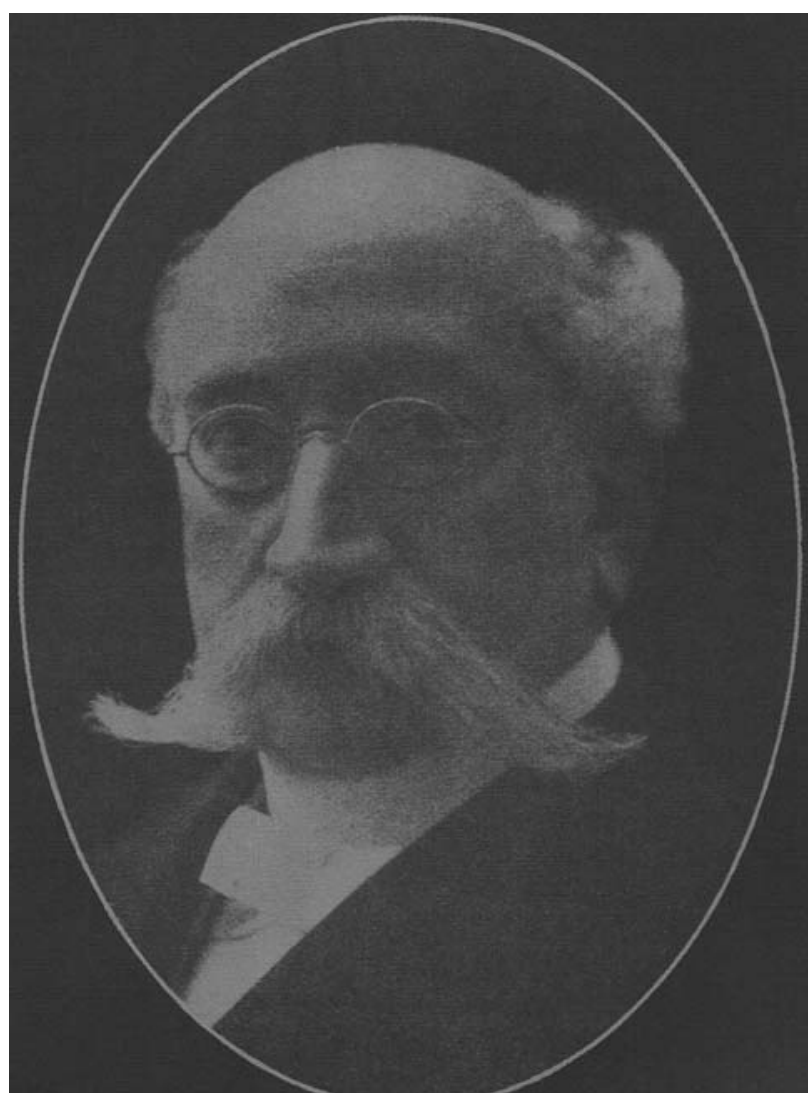

FIGURA 9. Dr. Salvador Cardenal Fernández (1852-1927) 


\section{Exposición Wniversal de tarcelona de 1888}

\section{Congresos}

DE

\section{Cienciàs Médicas}

\author{
DE BARGELONA
}

CELEBRADOS DEL 9 AL 15 DE SEPTIEMBRE DE 1888

por inielativa 7 con subroneion del

CONSEJO GENERAL DE LA EXPOSICIÓN UNIVERSAL DE BARCELONA

\section{II}

\section{CONGRESO MÉDICO}

\section{BARCELONA}

IMPRENTA DE J. BALMAS PLANAS Oalle del correo Viejo, 5 1889

FIGURA 10. Congreso de las Ciencias Médicas de Barcelona 1888. La Nefrectomía en Valencia, Dr. Más y Soler.

señala que hasta 1881 sólo se habían practicado en la ciudad tres laparotomías y que en 1894 llevaba realizadas diez nefrectomías por vía lumbar, con los diagnósticos de tuberculosis y de tumores y que, en caso de presentar una masa voluminosa, utiliza siempre la vía lumbo-abdominal extraperitoneal, año que publica Sarcoma voluminosote riñón. Nefrectomía ${ }^{16}$.

En Cataluña, el Dr. Carlos Perarnau, urólogo del Hospital de la Santa Cruz propone, en la Revista de Medicina y Cirugía Practica, en 1885, la nefrectomía sistemática en la anuria calculosa ${ }^{17}$ y el Dr. José Pagés y Puig (1863-1926), director del primer servicio de Vías Urinarias creado en Barcelona, en el Hospital del Sagrado Corazón, refiere un caso de nefrectomía en la misma publicación, en $1897^{18}$.

En septiembre de 1888, en el Congreso de las Ciencias Médicas celebrado en Barcelona con motivo de la Exposición Universal (Fig. 10), el Dr. Más y Soler (1847-1902), de Valencia, defiende la nefrectomía por vía abdominal frente a la lumbar en las operaciones por grandes tumoraciones renales y presenta dos enfermos intervenidos en ese mismo año por vía transperitoneal, diagnosticados uno de hidronefrosis litiásica supurada con una evolución séptica postoperatoria, que condujo al fallecimiento, y otro paciente que no presentaba infección, curado ${ }^{3}$.

Encontramos otro caso más de nefrectomía, realizada con éxito en un enfermo con litiasis renal, en agosto de 1887, sin especificar la vía, aunque presumimos que lumbar, por la formación y preparación francesa realizada en Montpellier del cirujano gran canario Dr. José Hernández Pérez (1856-1890), expuesto por el Dr. Chesa Ponce ${ }^{19}$.

Tabla 1. Cirujanos que realizaron nefrectomias por vía lumbar en España en el siglo XIX

\begin{tabular}{lll}
\hline 1874 & Dr. Rubio y Galí & Primera nefrectomía lumbar en Madrid, seguida de una segunda \\
1885 & Dr. Esquerdo & Una por ectopia renal, en Barcelona \\
1887 & Dr. Esquerdo & Una por quiste renal \\
1887 & Dr. Hernández & Una por litiasis, en Canarias \\
1889 & Dr. Rubio y Gali & Un caso en Madrid \\
1890 & Dr. Azcárreta & Dos casos por tuberculosis, en Barcelona \\
1890 & Dr. Salado Antonio & Un caso en Sevilla \\
1892 & Dr. Azcárreta & Cinco nefrectomías por litiasis \\
1892 & Dr. Rubio y Gali & Nefrectomía por pionefrosis \\
1894 & Dr. Cardenal & Diez casos por tuberculosis y tumor \\
1896 & Dr. Azcárreta & Cuatro casos por tumor renal \\
1897 & Dr. Pagés & Una en Barcelona \\
1898 & Dr. Azcárreta & Cinco nefrectomías por tuberculosis
\end{tabular}




\section{CONCLUSIÓN}

La introducción de la nefrectomía por vía lumbar en España fue tardía en relación con el resto de Europa, debido al atraso secular que arrastrábamos, únicamente cuando la técnica quirúrgica se encontraba plenamente desarrollada y las condiciones generales de los quirófanos en los Hospitales, tanto de anestesia como de asepsia y de utillaje quirúrgico, permitían poder abordar con mayor garantía de éxito la operación, se comenzó a realizar. Hemos localizado 31 extirpaciones renales extraperitoneales, en el último tercio del siglo XX, seis con el diagnóstico de litiasis, cinco de tumor, dos de tuberculosis, diez entre tumor y tuberculosis y una por pionefrosis, por ectopia y por quiste y cinco sin especificar diagnóstico, efectuadas por cuatro cirujanos generales y tres urólogos, tras el primer caso en Madrid, del introductor de la operación Dr. Federico Rubio y Galí, destacan en Barcelona los urólogos Drs. Víctor Azcárreta Colán y José Pagés y Puig, los cirujanos Drs. Esquerdo y Cardenal Fernández, el sevillano Dr. Antonio Solano y el urólogo canario Dr. José Hernández Pérez, como los pioneros de la intervención en nuestro país.

\section{REFERENCIAS}

1. Küss R, Gregoire W. Histoire illustrée de 1`Urologie, ed. Roger Dacosta, 1992, París.

2. Maganto Pavón E, Clemente Ramos. Introducción de la cirugía experimental en Urología, en Hitos en la Historia de la Urología III. Pulso edic. 2000, Madrid.

3. Más y Soler M. 1887. La nefrectomía en Valencia. Contribución al estudio de esta operación en España, Congreso de las Ciencias Médicas de Barcelona, pág. 924-931, impr. Balmas Planas, 1889, Barcelona.

4. Mollá Rodrigo R. Résultats éloignés de l`intervention chirurgicale dans les tumeurs malignes du rein, XIV Congrès International de Médicine, pág. 6-19, 1904, Madrid.
5. Guyon. Estadística quirúrgica de las nefrectomías, Rev Med y Cirug Prácticas, 1892;XXXI (391):337-348.

6. Sota y Lastra J. Consideraciones sobre la nefrectomía, Rev Med Cirug Prácticas. 1892;XVI(337):225-238; (379):337349.

7. Israel J. Citado por Rafael Mollá.

8. Riera Palmero J. Historia de la Urología Española; Actas del II Congreso Español de Historia de la Medicina, pág. 60, 1965, Salamanca.

9. Rubio y Galí F. Pionefrosis del riñón derecho. Extirpación, curación, Anales Real Academia Nacional de Medicina, Madrid, 1890;10:375-379.

10. Esquerdo A. Citado por Más y Soler.

11. Azcarreta Colán V. Dos casos de nefrectomía por tuberculosis renal con resultado satisfactorio, Rev Ciencias Médicas de Barcelona, pág. 847, 1890, Barcelona.

12. Ibíd. Cinco Nefrectomias por tuberculosis renal, Congreso Hispano Portugués de Cirugía y sus Especialidades Naturales, pág. 241, impr. Aguado, 1898, Madrid.

13. Ibíd. Serie de nefrectomias por cálculos, Rev Quirúrgica de Enfermedades de Vías Urinarias, 1899, Barcelona.

14. Ibíd. Resultados de la intervención quirúrgica de los tumores malignos de riñón, Rev Med y Cirug Prácticas, Madrid, 1901;LI:352-355.

15. Cardenal Fernández S. Manual práctico de cirugía antiséptica, pág. 811, 3 ${ }^{a}$ edic., ed. Biblioteca Ilustrada Espasa, 1894, Barcelona.

16. Ibid. Sarcoma voluminoso de riñón. Nefrectomía. Rev Med y Cirug Prácticas, Madrid, 1890;XVIII(482):433.

17. Perarnau C. Rev Med Cirug Prácticas, Madrid, 1890; XXVI: 659.

18. Pagés J. Rev Med y Cirug Prácticas, Madrid, 1887; XXI: 659.

19. Chesa Ponce N. La primera nefrectomía en Canarias, Actas Urol Esp 2004.

Correspondencia autor: Dr. M. Pérez Albacete

Servicio de Urología

Hospital Universitario Virgen de la Arrixaca

Ctra. Murcia-Cartagena, s/n - 30120 - El Palmar (Murcia)

Tel.: 968369779

E-mail autor: mariano.perez2@carm.es

Información artículo: Original - Historia de la urología

Trabajo recibido: septiembre 2007

Trabajo aceptado: octubre 2007 